\title{
A SATELLITE IMAGING MISSION PLANNING METHOD FOR FAST ANTARCTICA COVERAGE
}

\author{
Yaxin Chen ${ }^{1}$, Xin Shen ${ }^{1, *}$, Guo Zhang ${ }^{1}$, Tingting $\mathrm{Liu}^{2}$, ZeZhong Lu${ }^{1}$, Junfei Xu${ }^{1}$, Huilin Wang ${ }^{3}$ \\ ${ }^{1}$ State Key Laboratory of Information Engineering in Surveying, Mapping and Remote Sensing, Wuhan University, Wuhan 430079, \\ China \\ ${ }^{2}$ Chinese Antarctic Centre of Surveying and Mapping, Wuhan University, Wuhan 430079, China \\ ${ }^{3}$ Beijing Tracking and Communication Technology Research Institute, Beijing 100094, China \\ *Correspondence: xinshen@whu.edu.cn; Tel.: +86-186-2780-1540
}

Commission III, IV/III

KEY WORDS: Global warming, Antarctica imaging, Satellite mission planning, multi-objective optimization, Gaofen-3, LMOCSO algorithm

\begin{abstract}
:
Global warming has become one of the most prominent global issues, and Antarctic ice sheet is one of the indicator of global climate change. Satellite imagery has become an important means of monitoring the changes in Antarctic ice sheet. Due to the high overlap of satellite imaging swaths, the existing Antarctica images have the disadvantages of long period of imagery acquisition, large temporal difference among the mosaic images, and low utilization of satellite resource. This paper proposes a satellite imaging mission planning method for fast Antarctica coverage. First, the imaging time window is forecasted within the specified imaging time range to obtain all the visible time windows of the imaging satellite to Antarctica. Then, taking the selection of each time window and the satellite swing angle in each time window as decision variables, and the satellite attitude maneuver ability as constraint, an imaging mission model including two objective functions with minimum number of imaging time windows and the maximum coverage rate is established. To solving the proposed multi-objective optimization model, an improved real-binary hybrid LMOCSO (large-scale multi-objective optimization based on a competitive swarm optimizer) is proposed in this paper. Finally, a simulation experiment was performed using Gaofen-3 satellite to verify the effectiveness of the proposed method.
\end{abstract}

\section{INTRODUCTION}

A scientific report at the 2019 United Nations Climate Action Summit pointed out that from 2015 to 2019, the average global temperature increased by $1.1^{\circ} \mathrm{C}$ compared with the preindustrial era, and increased by $0.2^{\circ} \mathrm{C}$ compared to $2011-2015$. 2015-2019 is likely to be the hottest five years on record for humankind. The progress and severity of climate change far exceed the prediction and assessment ten years ago. The world is facing a critical climate tipping point (Hoegh-Guldberg et al., 2019). In 2018, "Future Earth" and "Earth League" released a report entitled "10 New Insights in Climate Science 2018" at the 24th Conference of the Parties to the United Nations Framework Convention on Climate Change (COP24). It is pointed out that many expected impacts of man-made climate change have appeared earlier, including:(1) the ice loss rate in Antarctica is accelerating, which is currently almost twice the latest assessment forecast of the United Nations Intergovernmental Panel on Climate Change (IPCC) 2014. (2) Satellite data confirm that sea level is rising. (3) Limiting climate warming to $1.5^{\circ} \mathrm{C}$ instead of $2^{\circ} \mathrm{C}$ can prevent the land currently inhabited by about 5 million people from being submerged (Pihl et al., 2018). The Antarctic span 14 million $\mathrm{km} 2$ (roughly twice the size of Australia), and its immense ice sheet is up to $4.8 \mathrm{~km}$ thick and contains $90 \%$ of the world's fresh water, enough to raise sea level by around 60 metres were it all to melt (Allison et al., 2017). The Antarctic Ice sheet/shelf is one of the important "indicators" to reflect global climate. Due to the harsh natural environment in Antarctica, satellite images have gradually become one of the important means to monitor changes in Antarctica, in addition to artificial Antarctic scientific research.

With the development of imaging satellite, the production of remote sensing image products for the whole Antarctica has been carried out many times in the world. The first remote sensing image mosaic of Antarctica was produced in 1987. It was made from 40 AVHRR images of NOAA series satellites from October 1980 to December 1983, with a spatial resolution of $1 \mathrm{~km}$ (Merson, 1989). The first mosaic image of Antarctica satellite imagery with high spatial resolution was taken by the Canadian RADARSAT-1 satellite. The radar imaged 100kilometer-wide swaths up to 2,500 kilo-meters long. The mosaic combined more than 4,500 overlapping images taken from September to October 1997 with a resolution of 25 meters (Jezek et al., 1998). In 2004, the mosaic of MODIS images in Antarctica was completed, and 260 MODIS images from November 2003 to February 2004 were used to produce mosaic images with $125 \mathrm{~m}$ and $750 \mathrm{~m}$ spatial resolution respectively (Bohlander et al., 2004). In 2007, NASA worked with the USGS, the National Science Foundation, and the British Antarctic Survey to map the world's first high-resolution, three dimensional, true color map of Antarctica (Bindschadler et al.,2008). The Landsat Image Mosaic of Antarctica (LIMA) is composed of more than 1100 images from the Landsat 7 satellite, acquired between 1999 and 2001. With a spatial resolution of $15 \mathrm{~m}$, it can clearly show the geographical

* Corresponding author 
characteristics of Antarctica, such as ice sheet, ice shelf, fastmoving ice flow, mountains. LIMA covers the entire continent except from the South Pole at 90 degrees south to 82.5 degrees south latitude, where Landsat has no coverage because of its near-polar orbit. The latest image of Antarctica is The Reference Elevation Model of Antarctica (REMA) (Howat et al.,2019) by the University of Minnesota and Ohio State University. REMA is a high resolution, time-stamped Digital Surface Model (DSM) of Antarctica at 8-meter spatial resolution. REMA is constructed from hundreds of thousands of individual stereoscopic Digital Elevation Models (DEM) extracted from pairs of submeter $(0.32$ to $0.5 \mathrm{~m})$ resolution Digital Globe satellite imagery, including data from WorldView-1, WorldView-2, and WorldView-3, and a small number from GeoEye-1, acquired between 2009 and 2017 。

Version 1 of REMA includes approximately $98 \%$ of the contiguous continental landmass extending to maximum of roughly $88^{\circ} \mathrm{S}$.

The above Antarctica images are important for monitoring Antarctica, but they also have some shortcomings: long period of imagery acquisition, large temporal difference among the mosaic images, and low utilization of satellite resource. In response to these shortcomings, this paper proposes a satellite imaging mission planning method for fast Antarctica coverage, which could develop a reasonable and efficient imaging solution. First, the imaging time window is forecasted within the specified imaging time range to obtain all the visible time windows of the imaging satellite to Antarctica. Next, taking the selection of each time window and the satellite swing angle in each time window as decision variables, the satellite attitude maneuver ability as a constraint, and the minimum number of imaging time windows and the maximum coverage rate of Antarctica as objective functions, a multi-objective optimization model is established. Then, use the improved realbinary hybrid LMOCSO to solve the model. Finally, a simulation experiment was performed using Gaofen-3 to verify that the method proposed could complete the imaging of Antarctica with as few satellite resources as possible in a specified time.

The remainder of the paper is organized as follows. In Section 2, the multi-objective optimization model and the real-binary hybrid LMOCSO algorithm are introduced; Experimental data, results and discussion are given in Section 3. Finally, the conclusions and the next work are drawn in Section 4.

\section{THE PROPOSED METHOD}

\subsection{Mission planning model for imaging satellite}

The mission planning model for Antarctica imaging is a multiobjective optimization model with two types of decision variables, two objective functions, and a constraint. The mathematical expression of the proposed mission planning model for fast Antarctica imaging is as follows:

- Decision variables:

Expression of decision variable 1:

$$
\mathrm{y}_{i}=\left\{\begin{array}{l}
1, \text { if the } i \text { th time window is selected } \\
0, \text { else }
\end{array}\right.
$$

Where decision variable $y_{i}$ represents the selection of the i-th time window. If it is selected, $y_{i}=1$; otherwise, $y_{i}=0 \cdot y_{i}$ is a binary variable.
Expression of decision variable 2:

$$
\mathbf{x}=\left(x_{1}, x_{2}, \cdots, x_{i}, \cdots, x_{n}\right)
$$

Where decision variable $\mathbf{x}$ represents the set of swing angles of imaging satellite. $x_{i}$ represents the swing angle of satellite imaging in the $\mathrm{i}$-th time window.

- Objective functions

Expression of objective function 1:

$$
\max f(\mathbf{x})=\frac{S_{\mathrm{cov}}(\mathbf{x})}{S_{o b j}}
$$

Where $S_{\text {cov }}(\mathbf{x})$ represents the imaging area of the satellite and $S_{o b j}$ represents the area of target. Objective function 1 ensures the maximum coverage rate of the imaging area. Expression of objective function 2:

$$
\min g\left(y_{i}\right)=\sum_{i=1}^{n} y_{i}
$$

Objective function 2 ensures the minimum number of time windows, that is, the minimum consumption of satellite resources.

- Constraint

Expression of the constraint:

$$
x_{\min } \leq x_{i} \leq x_{\max }
$$

where $x_{\min }$ and $x_{\max }$ are the minimum and maximum values of the satellite swing angle, respectively.

\subsection{Model solving based on real-binary hybrid LMOCSO}

Generally speaking, MOPs with more than 100 decision variables are known as large-scale multi-objective optimization problems (MOPs) (Ma et al., 2015) and (Zhang et al.,2016). In the Antarctica imaging task planning problem, there are more than 600 real decision variables and more than 600 binary decision variables, which is definitely a large-scale multiobjective optimization problem. Large-scale MOPs are much more difficult to be solved than those with a few decision variables, since the search space is exponentially related to the number of decision variables, which makes it impossible for multi-objective evolutionary algorithms (MOEAs) to explore the search space efficiently. To solving the proposed multiobjective optimization model, we proposes a real-binary hybrid LMOCSO based on LMOCSO proposed by Tian (Tian et al.,2019). The model solving process using the real-binary hybrid LMOCSO is as follows:

Step 1: Initialize the population.

Step 2: Generate uniformly distributed reference vector V, which will be used in environment selection.

Step 3: Calculate the fitness value of each particle using the shift based density estimation strategy calculated by Eq. (6)

$$
\operatorname{Fitness}(p)=\min _{q \in \mathbf{P} \backslash\{p\}} \sqrt{\sum_{i=1}^{M}\left(\max \left\{0, f_{i}(\vec{q})-f_{i}(\vec{p})\right\}\right)^{2}}
$$

where $f_{i}(\vec{p})$ denotes the $\mathrm{i}$-th objective value of particle $\mathrm{p}$ and $\mathrm{M}$ denotes the number of objectives. 
Step 4: Select loser and winner particles by the fitness value. Randomly select two particles $\{p, q\}$ from the current population. If fitness $(p)>\operatorname{fitness}(q), \mathrm{q}$ is the loser particle to be updated, and $\mathrm{p}$ is the winner, otherwise $\mathrm{p}$ is the loser particle and $\mathrm{q}$ is winner. Until all particles are judged.

Step 5: Update all loser particles. For real decision variables, the update strategy proposed by Tian (Tian et al.,2019) is adopted:

$$
\begin{aligned}
\vec{v}_{l}(t+1) & =r_{0} \vec{v}_{l}(t)+r_{1}\left(\overrightarrow{x_{w}}(t)-\overrightarrow{x_{l}}(t)\right) \\
\vec{x}_{l}(t+1) & =\vec{x}_{l}(t)+\vec{v}_{l}(t+1)+r_{1}\left(\vec{v}_{l}(t+1)-\vec{v}_{l}(t)\right)
\end{aligned}
$$

Where $\vec{v}_{l}(t)$ represents the velocity of the loser particle at the $\mathrm{t}$-th generation, $\overrightarrow{x_{w}}(t)$ represents the position of the winner particle at the $\mathrm{t}$-th generation, and where $\mathrm{r}_{0}$ and $\mathrm{r}_{1}$ are uniformly randomly distributed values in $[0,1]$.

For binary decision variables, the update strategy of velocity is the same as that of real decision variables, and the update strategy of position is based on sigmoid function:

$$
\begin{aligned}
& S\left(v_{l}\right)=\frac{1}{1+e\left(-v_{l}\right)} \\
& x_{l}=\left\{\begin{array}{l}
1 \text { if } \operatorname{rand}() \leq \mathrm{S}\left(v_{l}\right) \\
0 \text { else }
\end{array}\right.
\end{aligned}
$$

Where $S(\cdot)$ is sigmoid function, and $\operatorname{rand}()$ is uniformly randomly distributed values in $[0,1]$.

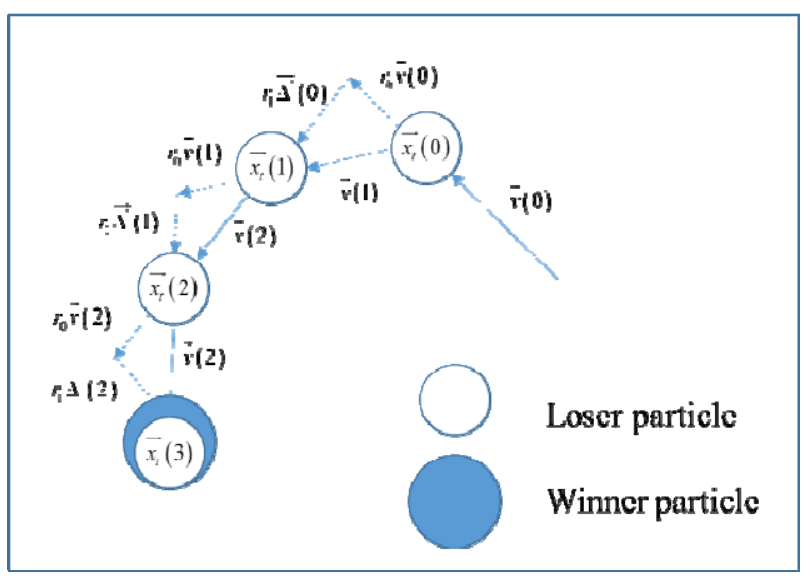

(a)The update strategy of real decision variables

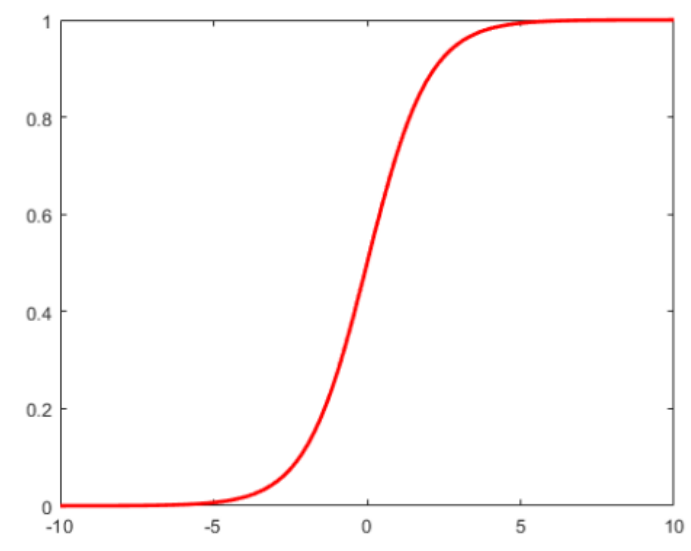

(b)The position update strategy of binary decision variables

Figure 1.The update strategy of decision variables

Step 6: Mutate all particles by polynomial mutation, which avoid model solving from getting into local optimums.

Step 7: Generate new population through environment selection. Associate each particle to its closest reference vector according to the angles between particles and reference vectors, then select one particle with the best angle-penalized distance.

Step 8: Iteration. If the iteration termination condition is satisfied, the final population is output, otherwise, step 3 is repeated.

\section{EXPERIMENTS AND DISCUSSIONS}

\subsection{Experimental satellite}

In this paper, Gaofen-3 satellite is used for imaging experiments in Antarctica. Gaofen-3 is China's first multi-polarization synthetic aperture radar (SAR) imaging satellite with a resolution of 1 meter and 12 imaging modes. The standard strip model is used for imaging experiment, which is one of modes suitable for snow cover and sea ice detection. The basic parameters of Gaofen-3 and its standard strip mode are shown in Table 1.

Table 1. The basic parameters of Gaofen-3 and its standard strip mode

\begin{tabular}{cc}
\hline Satellite & Gaofen-3 \\
\hline Launch time & Aug 10, 2016 \\
Orbit Type & Repeat sun-synchronous orbit \\
Orbital Altitude $(\mathrm{km})$ & 755 \\
Swing Ability & $17^{\circ}-50^{\circ}$ \\
Spatial resolution $(\mathrm{m})$ & 25 \\
Swath Width $(\mathrm{km})$ & 130 \\
Band & $\mathrm{C}$ \\
Polarization & Dual polarization \\
\hline
\end{tabular}

\subsection{Acquisition of imaging time window}

Antarctica covers an area of 14 million square kilometers and is located within $63^{\circ}$ south latitude. If the satellite has been imaging when passing through the Antarctica, the maximum imaging time can be more than 800 s. Although it can be imaged, it is difficult to perform the task due to the long imaging time. In order to obtain tasks that are easy to perform, we perform regional decomposition of the Antarctic. The Antarctic is decomposed into 9 sub-regions using the $6^{\circ}$ latitude difference, which guarantees that the length of each time window is between 100s and 110s, as shown in Figure 2. In order to get closer to the real situation, we think that the satellite can only image once when passing through the Antarctica, that is, only one of the 9 regions was imaged. In order to make Antarctica almost fully covered, we obtained a total of 663 time windows in 63 days from December 5, 2019 to February 5, 2020. There are too many time windows to list them one by one. Table 2 lists the number of time windows for each subregion. 


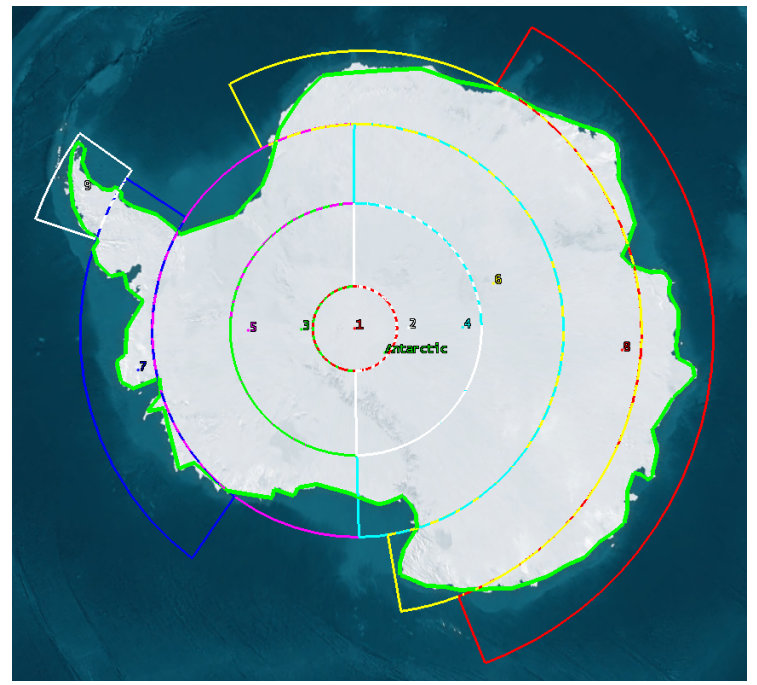

Figure 2. Antarctic decomposition for time window acquisition

Table 2. Regional scope and number of time windows

\begin{tabular}{ccc}
\hline NO. & $\begin{array}{c}\text { Subregion } \\
\text { (latitude;longtitude) }\end{array}$ & $\begin{array}{c}\text { Number of time } \\
\text { windows }\end{array}$ \\
\hline 1 & $-90^{\circ} \sim-87^{\circ} ;-180^{\circ}-180^{\circ}$ & 15 \\
2 & $-87^{\circ} \sim-81^{\circ} ; 0^{\circ}-180^{\circ}$ & 76 \\
3 & $-87^{\circ} \sim-81^{\circ} ;-180^{\circ}-0^{\circ}$ & 74 \\
4 & $-81^{\circ} \sim-75^{\circ} ; 0^{\circ}-180^{\circ}$ & 74 \\
5 & $-81^{\circ} \sim-75^{\circ} ;-180^{\circ}-0^{\circ}$ & 74 \\
6 & $-75^{\circ} \sim-69^{\circ} ;-27^{\circ} \sim 172^{\circ}$ & 146 \\
7 & $-75^{\circ} \sim-69^{\circ} ;-56^{\circ} \sim-143^{\circ}$ & 72 \\
8 & $-69^{\circ} \sim-63^{\circ} ; 30^{\circ} \sim 160^{\circ}$ & 115 \\
9 & $-69^{\circ} \sim-63^{\circ} ; 54^{\circ} \sim-70^{\circ}$ & 17 \\
\hline
\end{tabular}

\subsection{Experimental results}

In this paper, 663 time windows are used as model inputs, and the proposed multi-objective optimization model is solved using the real-binary hybrid LMOCSO to obtain an optimized Antarctic imaging scheme. Figure 3(a) shows the Antarctica imaging scheme with fixed swing angle (left swing $46^{\circ}$ ) before mission planning. Figure 3(b) shows the Antarctic imaging scheme optimized by the proposed model. Figure 4 and Figure 5 are detailed comparisons of Antarctica imaging scheme before and after mission planning. Region 9 was selected for detailed display because it is one of the Antarctica border regions, which are observed more frequently for sea ice change monitoring. Region 1 was selected for detailed display because the Region 1 is located near the pole and is difficult to observe due to the orbital inclination. Table 3 shows the number of time windows and coverage rate before and after mission planning for Region 1, Region 9, and Antarctica. Figure 6 represents the satellite imaging swing angle in different time windows. The red points represent the imaging swing angles of the satellite in the selected time window, and the blue points represent the swing angles of the satellite in the unselected time window. To imaging the pole region, the swing angle ranges from $-30^{\circ}$ to $50^{\circ}$, and the negative value means swing to the left. Figure 7 shows the change of coverage rate with the number of imaging time windows.

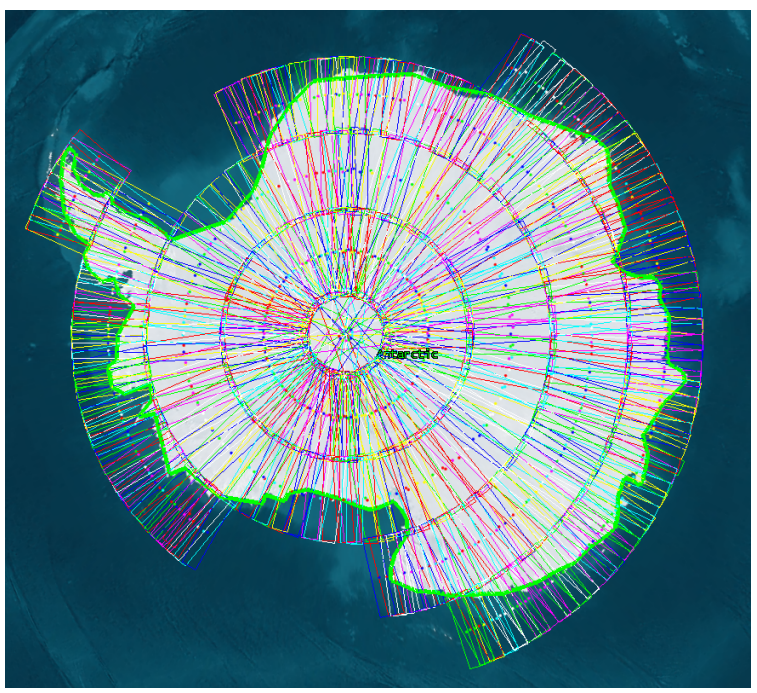

(a) Antarctic imaging scheme before mission planning

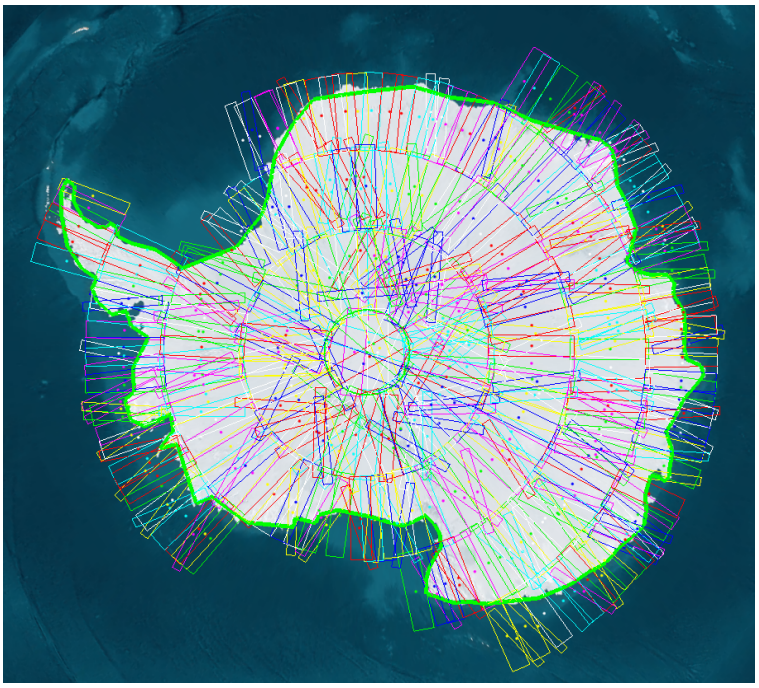

(b) Antarctic imaging scheme after mission planning

Figure 3. Antarctic imaging scheme

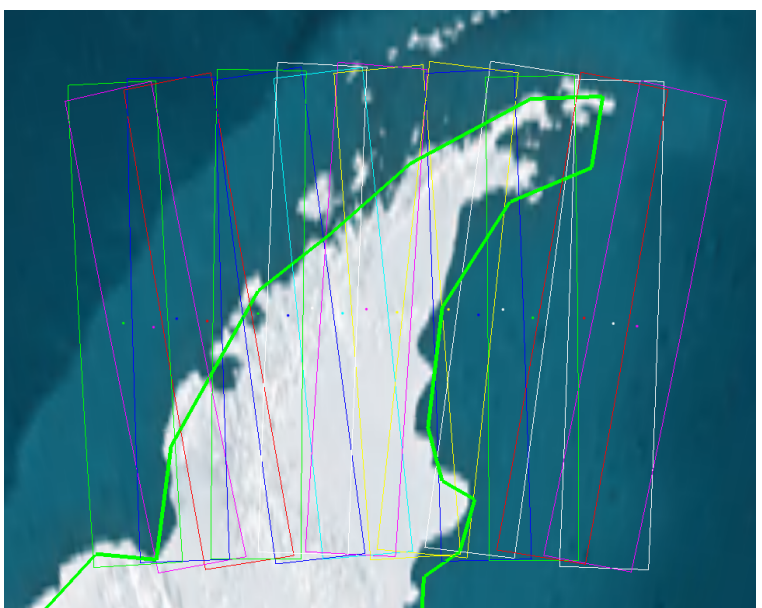

(a) Region 9 imaging scheme before mission planning 


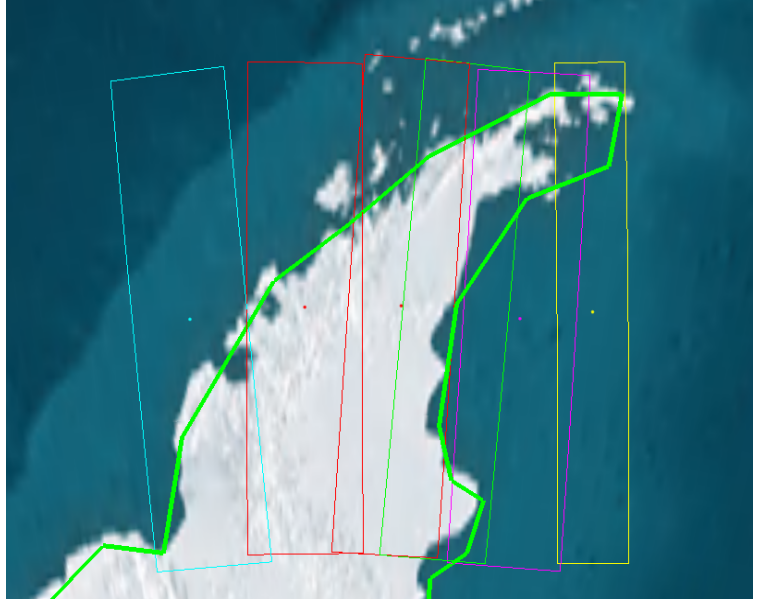

(b) Region 9 imaging scheme after mission planning

Figure 4. Detail 1 comparison of Antarctic imaging scheme

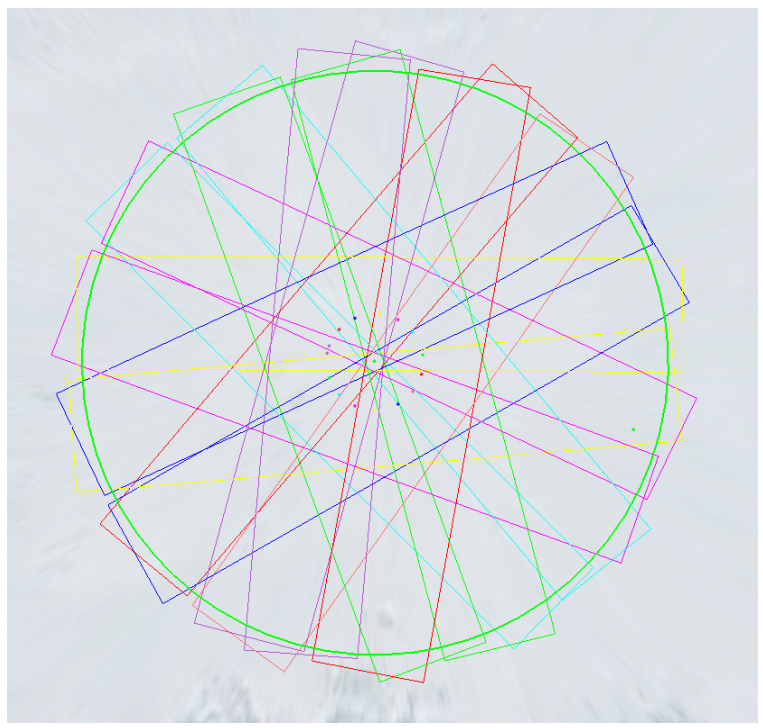

(a) Region 1 imaging scheme before mission planning

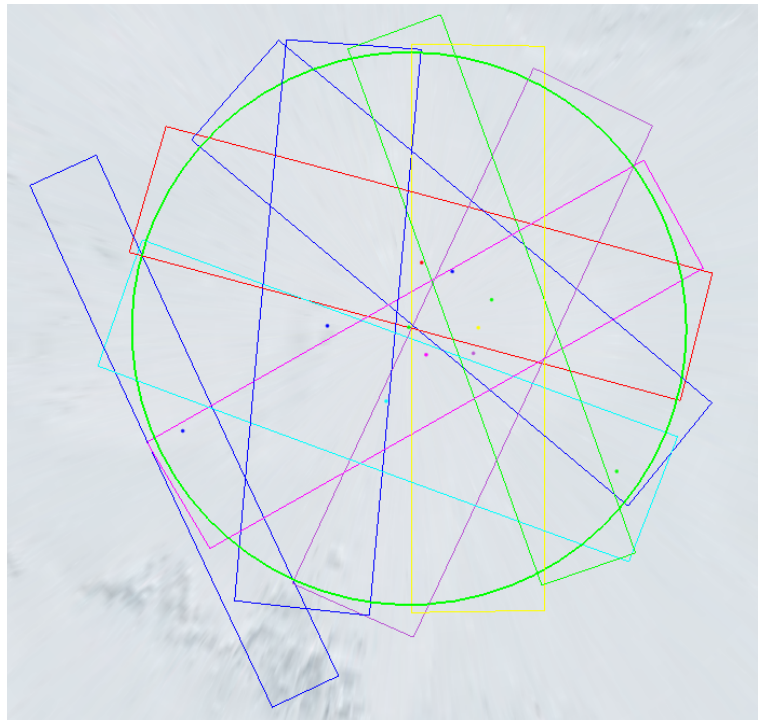

(b) Region 1 imaging scheme after mission planning

Figure 5. Detail 2 comparison of Antarctic imaging scheme
Table 3. Comparison of number of time windows and coverage rate before and after optimization

\begin{tabular}{lcccc}
\hline \multirow{2}{*}{ Region } & \multicolumn{2}{c}{ Before optimization } & \multicolumn{2}{c}{ After optimization } \\
\cline { 2 - 5 } & $\begin{array}{l}\text { Number } \\
\text { of time } \\
\text { windows }\end{array}$ & $\begin{array}{c}\text { Coverage } \\
\text { rate }\end{array}$ & $\begin{array}{l}\text { Number } \\
\text { of time } \\
\text { windows }\end{array}$ & $\begin{array}{c}\text { Coverage } \\
\text { rate }\end{array}$ \\
\hline Antarctica & 663 & $100 \%$ & 449 & $100 \%$ \\
Region 1 & 15 & $100 \%$ & 9 & $100 \%$ \\
Region 9 & 17 & $100 \%$ & 6 & $100 \%$ \\
\hline
\end{tabular}

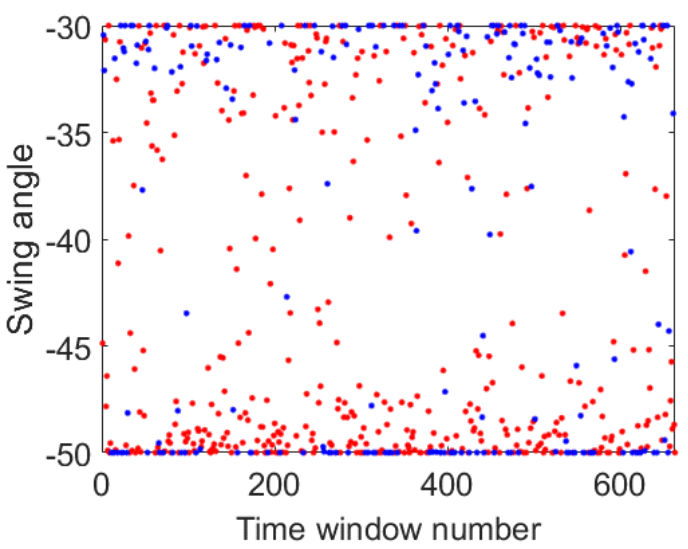

Figure 6. Swing angle in different time windows.

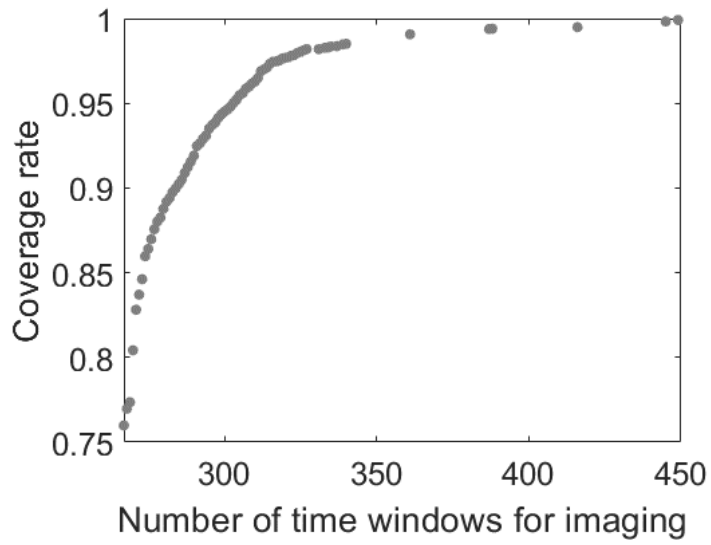

Figure 7 . The change of coverage rate with the number of time windows

\subsection{Discussion}

From the above experimental results, it can be seen that the proposed method for Antarctic imaging can greatly save satellite resources while maintaining the same coverage rate. From details 1 (Figure 4), it can be seen that region 9 that requires 17 imaging to be fully covered before optimization can be covered only 6 times after optimization, and the overlap rate of imaging strips is low. Compared with Region 9, the overlap rate of Region 1 (Figure 5) is higher. This is because Region 1 is located near the pole, which makes the imaging strips inevitably overlap. However, it has also been greatly improved compared to before optimization. Figure 6 shows the swing angle of satellite imaging in different time windows, and the selection of each time window, Red indicates that the time window is selected, and blue indicates that it is not selected. Based on these data, an Antarctic imaging scheme can be developed. The ratio of red points to blue points could also prove that satellite resources have been greatly saved. Figure 7 
shows the change of coverage rate with the number of imaging time windows. The data in Figure 7 shows that when 361 time windows are selected for imaging, the coverage can reach $99 \%$. This means that the remaining $1 \%$ of coverage takes 82 time windows, which increases the overlap rate. For example, the mission planning result of region 2 is not as good as region 9 , as shown in Figure 3(b). Users could choose a solution according to different needs.

Using as few satellites as possible to fully cover Antarctica can improve the mapping efficiency of Antarctica, reduce the temporal difference of mosaic images, and shorten the period of imagery acquisition. Efficiently acquiring Antarctic imagery has an important role in monitoring Antarctic changes and predicting future environmental changes, which could guide human activities to slow or prevent global warming.

\section{CONCLUSION}

This paper proposes a satellite imaging mission planning method for rapid Antarctica coverage. The objective functions of the proposed mission planning model includes: maximum coverage rate and minimum number of time window. Decision variables of the proposed model include the selection of the time window and swing angle of satellite imaging within each time window. Because the imaging planning of Antarctica is a large-scale problem in this paper, we use the real-binary hybrid LMOCSO algorithm to solve the proposed model. Finally, this paper takes Gaofen-3 as an example to conduct simulation experiments. By optimizing 663 time windows within 63 days, satellite resources can be greatly saved while fully cover Antarctica. The proposed method could become a reference for rapid Antarctic image production. In the next work, the imaging quality will be considered in the mission planning model.

\section{ACKNOWLEDGEMENTS}

We would like to thank the PlatEMO because they provide the MATLAB Code of the LMOCSO algorithm, which provides a good basis for our rapid implementation of the real-binary hybrid LMOCSO algorithm to solve the mission planning model. https://github.com/BIMK/PlatEMO

\section{REFERENCES}

Allison, I., Béland, M., Carlson, D., Qin, D., Sarukhanian, E., Smith, C., 2017. International Polar Year 2007-2008. Bulletin of the World Meteorological Organization.

Bindschadler, R., Vornberger, P., Fleming, A., Fox, A., Mullins, J., Binnie, D., Paulsen, S.J., Granneman, B., Gorodetzky, D., 2008. The Landsat image mosaic of Antarctica. Remote Sensing of Environment 112, 4214-4226.

Bohlander, J., Scambos, T., Haran, T., Fahnestock, M., 2004. A new MODIS-based mosaic of Antarctica: MOA, AGU Fall Meeting Abstracts.

Hoegh-Guldberg, O., Jacob, D., Taylor, M., Bolaños, T.G., Bindi, M., Brown, S., Camilloni, I., Diedhiou, A., Djalante, R., Ebi, K., 2019. The human imperative of stabilizing global climate change at 1.5 C. Science 365 , eaaw6974.

Howat, I.M., Porter, C., Smith, B.E., Noh, M.-J., Morin, P., 2019. The Reference Elevation Model of Antarctica. Cryosphere 13.
Jezek, K.C., Sohn, H., Noltimier, K., 1998. The RADARSAT Antarctic mapping project, IGARSS'98. Sensing and Managing the Environment. 1998 IEEE International Geoscience and Remote Sensing. Symposium Proceedings.(Cat. No. 98CH36174). IEEE, pp. 2462-2464.

Ma, X., Liu, F., Qi, Y., Wang, X., Li, L., Jiao, L., Yin, M., Gong, M., 2015. A multi-objective evolutionary algorithm based on decision variable analyses for multi-objective optimization problems with large-scale variables. IEEE Transactions on Evolutionary Computation 20, 275-298.

Merson, R., 1989. An AVHRR mosaic image of Antarctica. International Journal of Remote Sensing 10, 669-674.

Pihl, E., Canadell, J., Edenhofer, O., Gaffney, O., Rockström, J., Srivastava, L., Downy, C., Martin, M., Slavik, K., 2018. 10 New Insights in Climate Science 2018.

Tian, Y., Zheng, X., Zhang, X., Jin, Y., 2019. Efficient largescale multiobjective optimization based on a competitive swarm optimizer. IEEE Transactions on Cybernetics.

Zhang, X., Tian, Y., Cheng, R., Jin, Y., 2016. A decision variable clustering-based evolutionary algorithm for large-scale many-objective optimization. IEEE Transactions on Evolutionary Computation 22, 97-112. 